\title{
Pairing of one-dimensional Bose-Fermi mixtures with unequal masses
}

\author{
Matteo Rizzi ${ }^{1,2}$ and Adilet Imambekov ${ }^{3,4}$ \\ ${ }^{1}$ NEST-CNR-INFM and Scuola Normale Superiore, I-56126 Pisa, Italy \\ ${ }^{2}$ Max Planck Institut für QuantenOptik, Hans Kopfermann Strasse 1, D-85748 Garching, Germany \\ ${ }^{3}$ Department of Physics, Harvard University, Cambridge MA 02138, USA \\ ${ }^{4}$ Department of Physics, Yale University, P.O. Box 208120, New Haven, CT 06520-8120, USA
}

(Dated: November 4, 2018)

\begin{abstract}
We have considered one dimensional Bose-Fermi mixture with equal densities and unequal masses using numerical DMRG. For the mass ratio of K-Rb mixture and attraction between bosons and fermions, we determined the phase diagram. For weak boson-boson interactions, there is a direct transition between two-component Luttinger liquid and collapsed phases as the boson-fermion attraction is increased. For strong enough boson-boson interactions, we find an intermediate "paired" phase, which is a single-component Luttinger liquid of composite particles. We investigated correlation functions of such a "paired" phase, studied the stability of "paired" phase to density imbalance, and discussed various experimental techniques which can be used to detect it.
\end{abstract}

Recent developments in the cooling and trapping of atomic gases open exciting opportunities for experimental studies of interacting systems under well-controlled conditions. Using Feshbach resonances [1] and/or optical lattices [2, 3] it is possible to reach strongly interacting regimes, where correlations between atoms play a crucial role. The effect of interactions is most prominent for low-dimensional systems, and recent experimental realization [4, 5] of a strongly interacting Tonks-Girardeau (TG) gas of bosons opens new perspectives in experimental studies of strongly interacting systems in 1D. In particular, one can experimentally study the behavior of Bose-Fermi (BF) mixtures [6] in 1D. Due to the lack of candidate systems in traditional solid state experiments, this topic did not attract much theoretical attention until recently. By now, properties of 1D BF mixtures have been investigated using mean-field approximation [7], Luttinger liquid (LL) formalism [8, 9, 10, 11], T-matrix approximation [12], exact solutions [13], and numerical methods [14, 15, 16, 17]. The mean-field approximation is unreliable in 1D, the LL approach describes the phase diagram in terms of universal parameters which are hard to relate to experimentally controlled parameters, and the exact solution is restricted only to a certain region of parameter space. Most of the numerical work so far considered BF mixtures in optical lattices with fillings of the order of unity. In such a regime the analysis of the phase diagram is complicated, since the physics of the Mott transition plays an important role. In this article, we are mainly interested in the properties of 1D mixtures without optical lattice, for the regime of parameters directly relevant to current $\mathrm{K}-\mathrm{Rb}$ experiments [18]. Our main result is summarized in Fig. [1. For K-Rb mixture with equal density of bosons and fermions, we find the "pairing" phase for moderate interaction strengths. Such a phase was previously discussed in Ref. [8] for the case of almost equal masses and strong interactions based on LL formalism, but its relevance for current experiments with $\mathrm{K}-\mathrm{Rb}$ mixtures has not been addressed before. We note that the existence of "pairing" discussed in this article does not require the presence of commensurate optical lattice, as in Ref. [19].

The general Hamiltonian of a 1D BF mixture is given by

$$
\mathcal{H}=\int_{0}^{L}\left(\frac{\hbar^{2}}{2 m_{b}} \partial_{x} \Psi_{b}^{\dagger} \partial_{x} \Psi_{b}+\frac{\hbar^{2}}{2 m_{f}} \partial_{x} \Psi_{f}^{\dagger} \partial_{x} \Psi_{f}\right) \mathrm{d} x+\int_{0}^{L}\left(\frac{1}{2} g_{b b} \Psi_{b}^{\dagger} \Psi_{b}^{\dagger} \Psi_{b} \Psi_{b}+g_{b f} \Psi_{b}^{\dagger} \Psi_{f}^{\dagger} \Psi_{f} \Psi_{b}\right) \mathrm{d} x,
$$

where $\Psi_{b}, \Psi_{f}$ are boson and fermion operators, $m_{b}, m_{f}$ are the masses, and $g_{b b}, g_{b f}$ are boson-boson and boson-fermion interaction strengths. Well away from confinement induced resonances [20], 1D interactions are given by

$$
g_{b b}=2 \hbar \omega_{b \perp} a_{b b} \text { and } g_{b f}=2 \hbar \frac{\omega_{b \perp} \omega_{f \perp}\left(m_{b}+m_{f}\right)}{\omega_{b \perp} m_{b}+\omega_{f \perp} m_{f}} a_{b f},
$$

where $\omega_{b \perp}, \omega_{f \perp}$ are transverse confinement frequencies, and $a_{b b}, a_{b f}$ are 3D scattering lengths. $g_{b b}$ and $g_{b f}$ can be controlled by changing transverse confining frequencies, or by varying scattering lengths using Feshbach resonances [1]. For a K-Rb mixture in the absence of magnetic field, $a_{b b}>$ and $a_{b f}<0$, so in this article we will study the regime when boson-fermion interaction is attractive and boson-boson interaction is repulsive. The phases of Hamiltonian (10) in the most general case depend on four dimensionless parameters, which we choose to be $m_{b} / m_{f}, n_{b} / n_{f}$,

$$
\gamma_{b b}=\frac{m_{b} g_{b b}}{\hbar^{2} n_{b}} \text { and } \gamma_{b f}=\frac{\sqrt{m_{b} m_{f}} g_{b f}}{\hbar^{2} \sqrt{n_{b} n_{f}}} .
$$

Here $n_{f}, n_{b}$ are fermion and boson densities, and $\gamma_{b b}$ and $\gamma_{b f}$ are dimensionless interaction parameters. Similar to LiebLiniger model [21], strongly interacting regime corresponds to $\gamma_{b b},\left|\gamma_{b f}\right| \gg 1$. If $\gamma_{b b}<0$ (attractive bosons), the system 


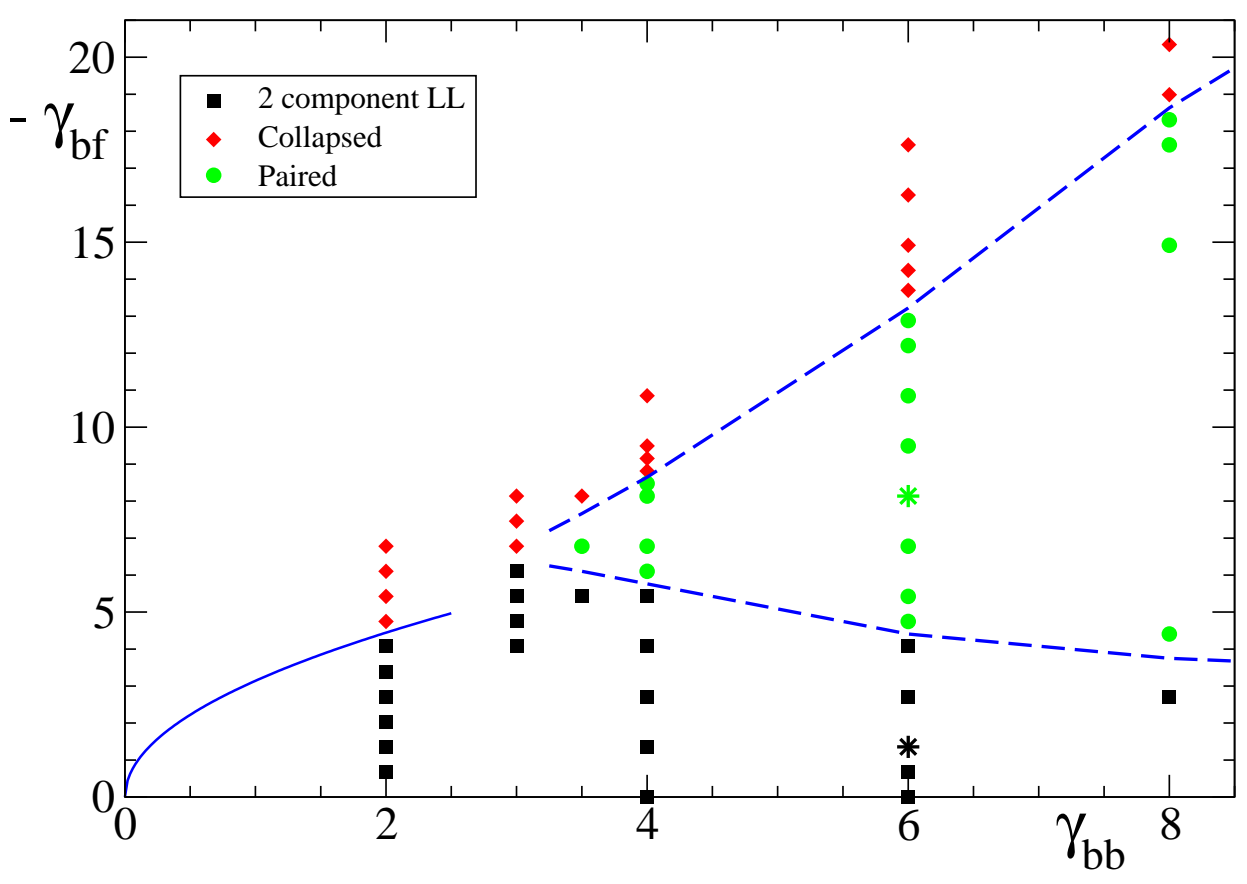

FIG. 1: Phase diagram for K-Rb mixture with equal density of bosons and fermions. $\gamma_{b b}$ and $\gamma_{b f}$ are defined by Eq. (2), and we show here the attractive side $\left(\gamma_{b f}<0\right)$. Different symbols and colors identify the three different phases involved: squares are in the two-component Luttinger liquid (LL) state, diamonds are in the collapsed state, whereas circles stand for the paired phase we found in between. Sample points, considered in Figs. 2, 3 , and 4 are identified by stars. Solid line is the mean-field prediction [7], which should be good for small $\gamma_{b b}$ and matches the data for smallest $\gamma_{b b}$ available. Dashed lines are tentative boundaries between the different phases. Notice the existence of a threshold in $\gamma_{b b}$ to get the paired phase.

is always unstable towards boson collapse. For $\gamma_{b b}>0$ the system can still collapse for $\gamma_{b f}<0$, or phase separate for $\gamma_{b f}>0$ [8]. If these two scenarios are not realized and densities of bosons and fermions are incommensurate, then from LL theory [8, 9, 10] one expects a two-component LL, with power law decay of all correlations. If densities are commensurate, one can expect [8, 11] a nontrivial pairing, resulting in the exponential decay of certain correlation functions and in the opening of the gap. In what follows we will concentrate on the latter case for an experimentally relevant K-Rb mixture, so we will fix $n_{b} / n_{f}=1, m_{b} / m_{f}=87 / 40$ and consider $\gamma_{b f}$ negative.

For numerical purposes we consider the discretized version of Hamiltonian (1) to be an open boundary chain with unity lattice constant and $L$ sites. Similar to the Lieb-Liniger model being a low filling fraction limit of Bose-Hubbard model [22], Hamiltonian (11) is the low filling fraction limit of the following lattice Hamiltonian:

$$
\mathcal{H}_{L}=-\sum_{i=1}^{L-1}\left(t_{b}\left(b_{i}^{\dagger} b_{i+1}+H . c .\right)-t_{f}\left(f_{i}^{\dagger} f_{i+1}+H . c .\right)\right)+\sum_{i=1}^{L}\left(\frac{U_{b b}}{2} b_{i}^{\dagger} b_{i}\left(b_{i}^{\dagger} b_{i}-1\right)+U_{b f} b_{i}^{\dagger} b_{i} f_{i}^{\dagger} f_{i}\right) .
$$

We note that many different lattice Hamiltonians give continuum Hamiltonian (11) in the low density limit, and the choice of lattice Hamiltonian is not unique. For low fillings $\nu_{b}$ and $\nu_{f}$, dimensionless interaction parameters are given by

$$
\gamma_{b b} \approx \frac{U_{b b}}{2 t_{b} \nu_{b}} \text { and } \gamma_{b f} \approx \frac{U_{b f}}{2 \sqrt{t_{b} \nu_{b} t_{f} \nu_{f}}}
$$

Most of our simulations were performed at densities $\nu \simeq 1 / 4$, but some of the results were checked for $\nu \simeq 1 / 8$. The fact that we use finite filling fractions only slightly changes Eq. (4), but does not affect the phase diagram qualitatively. We stress here that our results were checked also against commensurability effects by testing them at filling fractions $\nu=23 / 96$ and $\nu=25 / 96$ too. Thus we can safely say that pairing effects are not depending on the particular $\nu$ value chosen. The expectation values $\langle\ldots\rangle$ of one- and two-body operators over the ground state of $\mathcal{H}_{L}$ have been evaluated by means of the DMRG method [23], which provides a practically exact solution for any value of the couplings and allows one to measure correlation functions with both statistics on the equal footing. We used up to $L=96$ chains, with local dimension $d=10$ (up to 4 bosons per site) and truncation up to $m=256$ states. Discarded probabilities 
amount to less than $\varepsilon=5 \cdot 10^{-7}$. Small values of $\gamma_{b b}$ with weak interparticle interactions, however, are not easy to study with this method, since high occupation number for bosons should be taken into account. We thus resorted to mean-field predictions in this area.

Local density profiles were measured and are plotted in Fig. 2 for two sample points (corresponding to stars in Fig. 11). In our simulations we calculated the following correlation functions: bosonic Green function $G_{b}(i, j)=\left\langle b_{i}^{\dagger} b_{j}\right\rangle$, fermionic Green function $G_{f}(i, j)=\left\langle f_{i}^{\dagger} f_{j}\right\rangle$ and "pairing" correlation function $G_{b f}(i, j)=\left\langle\Delta_{i}^{\dagger} \Delta_{j}\right\rangle=\left\langle b_{i}^{\dagger} f_{i}^{\dagger} f_{j} b_{j}\right\rangle$, where the "pairing" operator is described by $\Delta_{j}=f_{j} b_{j}$. Their values have been plotted in Fig. 3 for the same sample points as before. Fourier transforms of bosonic and fermionic Green functions give respective momentum distributions, while Fourier transform of $G_{b f}(i, j)$ is related to momentum distribution of composite particles. Such momentum distributions are shown in Fig. 4. In order to calculate them, it is crucial to choose the properly defined free-particle eigenmodes due to open boundaries $\phi_{k}(j)=\sqrt{2 /(L+1)} \sin (k j)$ with $k=n \frac{\pi}{L+1}(n=1, \ldots, L)$. In addition to these correlation functions, density-density correlations $D_{\alpha, \beta}=\left\langle n_{\alpha}(i) n_{\beta}(j)\right\rangle-\left\langle n_{\alpha}(i)\right\rangle\left\langle n_{\beta}(j)\right\rangle$ were measured as well. Here $\alpha$ and $\beta$ can take any value from $\{b, f\}$. For finite size simulations, subtraction of the non-connected part is necessary, since open boundary conditions give density profiles with Friedel oscillations. Examining of density correlations without this substraction could lead to misinterpretation of results.

To extract the long-range behavior of correlation functions, we restricted the analysis to the regions far away from the boundaries. In order to check whether a certain correlation function has a power-law or exponential decay, it is sufficient to test the simplest power-law and exponential forms

$$
\left.G_{*}(x)=\begin{array}{c}
A_{*} e^{-x / d_{*}} \\
B_{*}|x|^{-\alpha_{*}}
\end{array}\right\} \sin \left(\omega_{*} x+\varphi_{*}\right)
$$

where the oscillating term is absent for the pure bosonic Green function. The fermionic oscillation frequency is correctly given by the fermionic density $\omega_{f}=\pi \nu_{f} \simeq \pi / 4$. The exact value is given by the density in the system bulk, which is slightly larger due to open boundary conditions. For convenience, let us introduce the Luttinger parameters $K_{b}, K_{f}$ and $K_{b f}$, which are related to $\alpha_{b}, \alpha_{f}$ and $\alpha_{b f}$ in Eq.(5) as $\alpha_{b}=1 /\left(2 K_{b}\right), \alpha_{f(b f)}=\left(K_{f(b f)}+1 / K_{f(b f)}\right) / 2$. Equation (5) gives the asymptotic form of the correlation functions in the thermodynamic limit far from the boundaries. To quantitatively extract the Luttinger parameters $K_{*}$ for finite $L$, one has to take into account carefully the effects of open boundary conditions (OBCs). We refer the reader to the detailed analysis of Ref. 24] and recall here that $G_{*}(i, j)$ will depend on chord functions $d_{L}(x)=d(x \mid 2(L+1))=(2(L+1) / \pi) \sin (\pi x / 2(L+1))$ of all $2 i, 2 j, i-j, i+j$. The form of the correlation function which needs to be fitted to extract Luttinger parameters are given by $\left(G_{b f}\right.$ is modified in the same way as $G_{f}$ )

$$
\begin{aligned}
G_{b}(i, j) \propto & {\left[d_{L}(2 i) d_{L}(2 j)\right]^{\frac{1}{4 K_{b}}}\left[d_{L}(i+j) d_{L}(i-j)\right]^{-\frac{1}{2 K_{b}}}, } \\
G_{f}(i, j) \propto & {\left[d_{L}(2 i) d_{L}(2 j)\right]^{-\frac{1}{4}\left(K_{f}-\frac{1}{K_{f}}\right)}\left[d_{L}(i+j) d_{L}(i-j)\right]^{-\frac{1}{2}\left(K_{f}+\frac{1}{K_{f}}\right)} } \\
& \left\{A_{0}\left[d_{L}(i+j)\right]^{K_{f}}(-1)^{\operatorname{sign}(i-j)} \sin \left(\pi \omega(i-j)+\varphi_{0}\right)+A_{1}\left[d_{L}(i-j)\right]^{K_{f}} \sin \left(\pi \omega(i+j)+\varphi_{1}\right)\right\} .
\end{aligned}
$$

We start our tour around the phase diagram by looking at small attractions between bosons and fermions. The sample point $\gamma_{b b}=6.0$ and $\gamma_{b f}=-1.36$ is considered in the first panels of Figs. 2 and 3 Looking at density profiles in Fig. 2, one can notice that both the atomic species spread out around the whole lattice, exhibiting Friedel oscillations due to hard walls at the boundaries. Wings are cut off for the sake of plot clearness. Due to attraction between bosons and fermions such oscillations are in-phase, but the values of the two densities differ on the order of the second digit. Coming to correlation functions (illustrated in Fig. 3, first panel), one can easily recognize a power-law decay for both the bosonic Green function $G_{b}(i, j)$ (black circles) and the (oscillating) fermionic Green function $G_{f}(i, j$ ) (red squares). "Pairing" correlation function $G_{b f}(i, j)$ exhibits power-law behavior as well. Thus this phase is a twocomponent Luttinger liquid. Such phase has two gapless sound modes, and all correlation functions have algebraic decay.

Increasing the interspecies attraction for small $\gamma_{b b}$ will lead to a collapse. More precisely, bosons form a small region with high density where fermions will be attracted up to Pauli-allowed density $\nu=1$. Existence of such maximal density is an artifact of our lattice discretization, and is not expected in the absence of a lattice. According to mean-field theory [7], the first order phase transition between the two component LL and the collapsed phase should take place as boson-fermion attraction is increased for any value of the boson-boson interaction. Within meanfield theory transition line is given by $\gamma_{b f}^{2}=\gamma_{b b} \pi^{2}$, and it is shown in Fig. 1 as a solid line. The result of mean field calculation agrees well with the data set for smallest $\gamma_{b b}$ considered. For large $\gamma_{b b}$, mean-field calculation is not expected to give an adequate description of the system, and for sufficiently large $\gamma_{b b}$ and attractive fermion-boson term $\gamma_{b f}<0$ the system belongs to a third intermediate phase, see Fig. 1 for a sketch. The population distributions and the correlations for a sample point in this intermediate region of parameters are plotted in the second panels of Figs. 2 

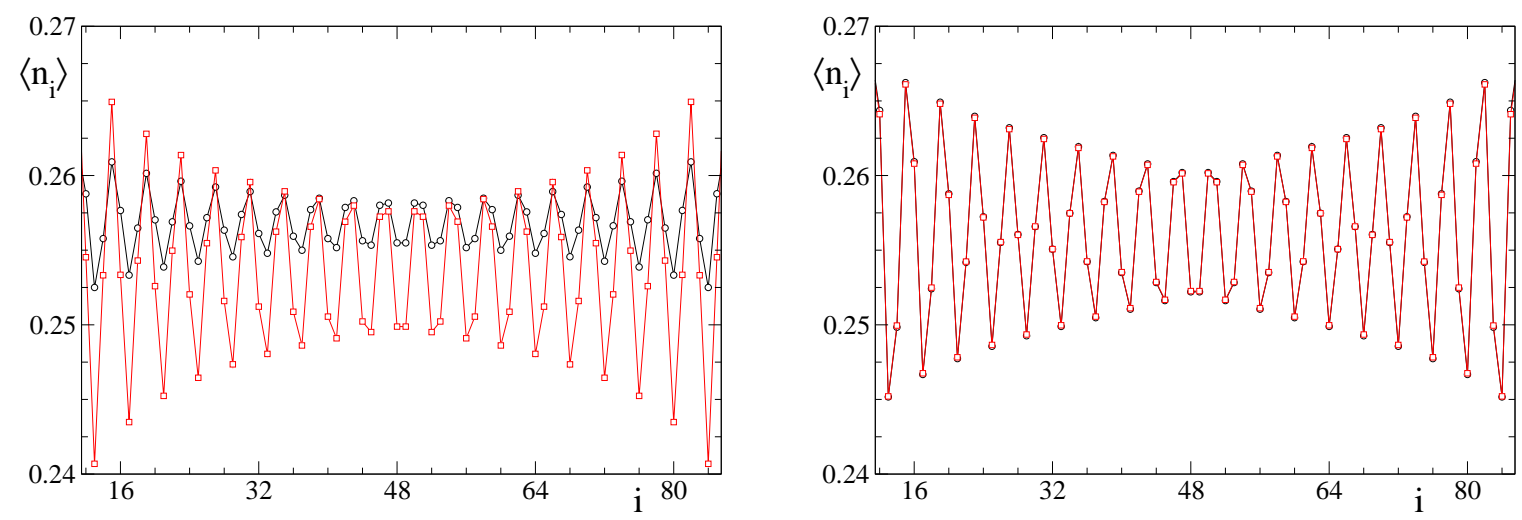

FIG. 2: Density profiles: black circles are bosons, red squares fermions. In the first panel we show results for a typical point in the usual mixed phase $\left(\gamma_{b b}=6.0, \gamma_{b f}=-1.36\right)$. Both the species are spread around the chain and no pinning is evident between densities. In the second panel, the "locking" of densities for the paired phase is evident. Right panel corresponds to the values $\gamma_{b b}=6.0, \gamma_{b f}=-8.14$. Friedel oscillation frequency is in both cases given, as expected, by $\omega_{*}=\pi \nu_{*}$ with $\nu_{*} \simeq 1 / 4$ being the species' density in the bulk of the system.

and 3. A strong locking of one density profile on top of the other is the most striking feature in Fig. 2(b). Indeed, not only the Friedel oscillations are in phase as they were in Fig. 2(a), but the difference between boson and fermion local densities is bounded to be less than $10^{-4}$ in the bulk, which is two orders of magnitude smaller than in the case of two-component LL. Furthermore, the strong locking of the two densities suggest that a composite particle made by a boson and a fermion, polaron, could be the new elementary object to look at. In the second panel of Fig. 3 all three types of correlation functions are plotted: $G_{b}$ (black circles), $G_{f}$ (red squares) and $G_{b f}$ (green diamonds). In contrast to two-component LL, single species Green functions clearly exhibit an exponential decay with a correlation length of few sites. However, the "pairing" correlations $G_{b f}$ still decay slowly as a power-law. Taking the open boundary conditions into account as described before, we get a Luttinger parameter $K_{b f}=0.95 \pm 0.02$. As shown in Fig. 4 such a dramatic change in the decay properties of correlation functions is witnessed by momentum distributions of the two species (and the composite one). Indeed, the Fermi step of individual fermionic atoms is no more there as it is in the case of two-component LL, and also the once peaked Bose distribution is considerably spread out now. In contrast, we highlight that momentum distribution of paired composite particles clearly exhibit a Fermi step around $k_{b f}=\pi / 4$ consistently with the filling. Thus, this phase can be understood as the "paired" phase of bosons and fermions. The existence of such a "paired" phase has been predicted in Ref. [8] based on LL theory arguments for mixture with equal masses for large $\gamma_{b b}$. Indications of the existence of such phases have also been briefly presented in Ref. [17], but the phase diagram has not been studied in detail. Figure 1 presents the phase diagram for the K-Rb mixture, and shows that "paired" phase can be realized for moderately strong Bose-Bose interactions. Boundaries between different phases were determined comparing algebraic and exponential fits of single species correlation functions, and by observing the "locking" of one density profile on the top of the other.

Looking at density-density correlations, one can address another distinctive feature of "paired" phase. As predicted in Ref. [8], oscillating part of all three density correlation functions $D_{\alpha, \beta}(x)(b-b, f-f, b-f)$ decay with distance with the same algebraic exponent

$$
\left.D_{*}(x)\right|_{2 \pi \nu} \sim|x|^{-r} \sin (\omega x+\varphi) .
$$

The frequency of oscillations is twice the particle density $\omega \simeq 2 \pi \nu \simeq \pi / 2$. As pointed out by Ref. [8], exponent $r$ should be intimately related to the Luttinger parameter for paired particles $K_{b f}$, i.e. $r=2 K_{b f}$ (we note that in our notations $K_{b f}=K_{+} / 2$, where $K_{+}$in defined in Ref. [8]). Thus parameter $K_{b f}$ can be extracted independently from $D_{\alpha, \beta}$, using the fitting procedure which takes OBC into account. We checked that all density-density correlations decay with the same exponent, and extracted value of $K_{b f}$ equals $0.97 \pm 0.02$. This is in good agreement with the pairing correlation fits (see before) which give the value $0.95 \pm 0.02$. Both these derivations have been checked for scaling with respect to both the system size $L$ (from 48 to 128) and the DMRG truncation parameter $m$ (up to 320 , discarded down to $<10^{-8}$ ). The values of $K_{b f}$ has been thus confirmed to survive to the thermodynamical limit. Based on all evidence, we can unambiguously state that we have shown the existence of the "paired" phase predicted by Cazalilla and Ho [8], even with moderate interactions and unequal masses of the two atomic species for the experimentally relevant case of $\mathrm{K}-\mathrm{Rb}$ mixture. Such a phase survives up to the thermodynamical limit with an almost unchanged exponent near 1 . We recall here that the universal prediction of unity value holds only at the 
transition point itself which, on the other hand, is difficult to be precisely addressed due to its BKT nature.
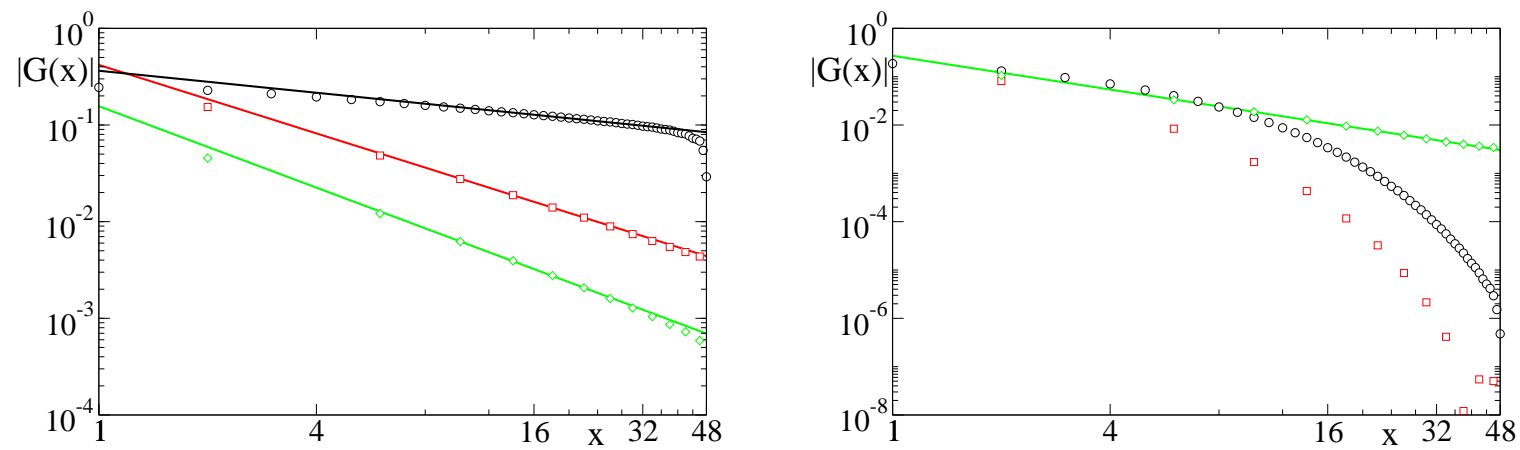

FIG. 3: Correlation functions: same sample points as in Fig. 2, same color code. For clarity, oscillations are not shown and only the decay of the envelope functions is presented. $G(x)$ means here $G(L / 2, L / 2+x)$. Green diamonds stand for composite particles created by $\Delta^{\dagger}=b^{\dagger} f^{\dagger}$. In the first panel (two-component Luttinger liquid state) all the three types of correlation functions exhibit an algebraic decay. For bosons $G_{b}(x) \propto x^{-1 /\left(2 K_{b}\right)}$ with $K_{b}=1.45 \pm 0.05$, whereas for fermions $G_{f}(x) \propto x^{-1 /\left(2 K_{f}\right)-K_{f} / 2}$ with $K_{f}=0.98 \pm 0.02$. On the other hand, for "paired" phase (second panel) only $G_{b f}$ shows an algebraic decay $\simeq x^{-1 /\left(2 K_{b f}\right)-K_{b f} / 2}$ with the Luttinger parameter $K_{b f}=0.95 \pm 0.02$, while $G_{b}$ and $G_{f}$ decay exponentially with distance.
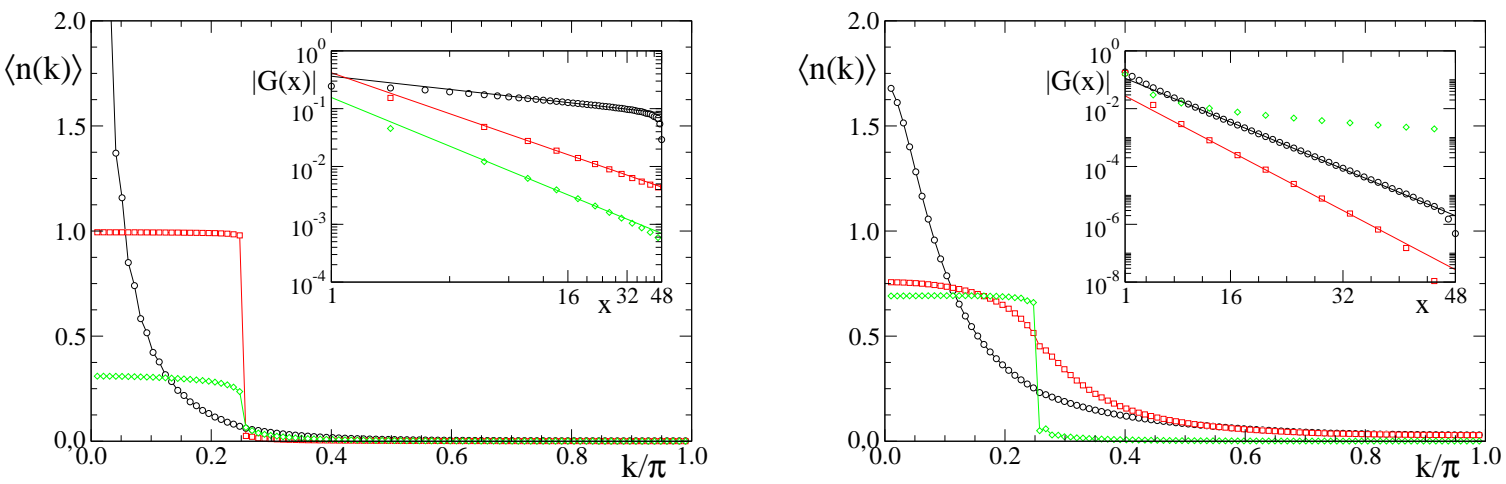

FIG. 4: Momentum distributions: same sample points and same color code of correlations as in Fig. 3. In the first panel, data for the two-component Luttinger liquid phase, where a Fermi surface for individual fermions and a tightly peaked distribution for bosons can be clearly seen. In the second panel, momentum distributions in the "paired" phase. We highlight the washing out of the Fermi surface for individual ${ }^{40} \mathrm{~K}$ and the wide broadening of the bosonic ${ }^{87} \mathrm{Rb}$ distribution. On the contrary, a sharp step-like feature in the composite particles' mode occupation is present around $\pi / 4$, indicating the algebraic decay of "pairing" correlation function $G_{b f}(x)$. Bosonic and fermionic particle correlations decay exponentially (see the inset, which is the same data as in the second panel of Fig. 3 but in log-linear scale).

The stability of "paired" phase against the population imbalance between the two species was also briefly studied (see Fig. 5). It turns out that for small enough density differences, the "locking" of the densities survives in the sides of the box, whereas in the middle a peak or a hole arises in the bosonic profile. The system phase separates into the region which exhibits "pairing" and the region with unequal densities. For larger density imbalance, the system becomes unstable to collapse, and the "paired" phase is washed out. This example qualitatively illustrates that the "paired" phase can be observed even if the densities of bosons and fermions are not exactly equal, but the imbalance is smaller than some threshold. More detailed studies of phases with unequal densities lie beyond the scope of this work.

Let us now concentrate on possible experimental techniques to detect the "paired" phase. One notable feature, which distinguishes the "paired" phase from the two-component LL phase, is the presence of the gap for out-of-phase density modes. Deep in the "paired" phase, the energy scale for the gap is set by Fermi energy $\sim \pi^{2} \hbar^{2} n^{2} /(2 m)$. The presence and the size of the gap can be measured using RF spectroscopy [25, 26]. Energies of sound modes can be measured using Bragg scattering [27], and the "paired" phase has only one sound mode, as opposed to two-component LL phase, which has two modes. Disappearance of the out-of-phase sound mode also affects qualitatively the response of the system to the variations of the external potential, since out-of-phase collective mode 

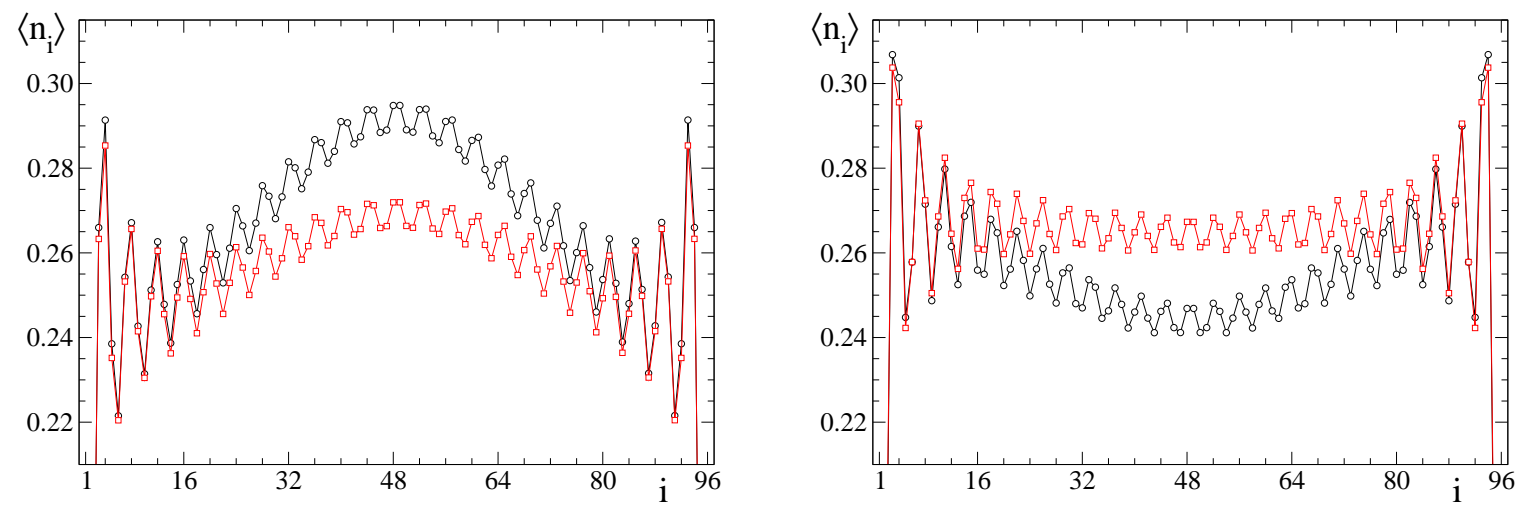

FIG. 5: Density profiles in the presence of an extra boson (first panel) or fermion (second one) with respect to filling $1 / 4$ in the paired region (same settings as Fig. 2). The two species' profiles retain their "pairing" in the wings and exhibit a peak or a hole in the bosonic density in the center of the box. For larger density imbalance, the system becomes unstable to collapse.

in external trap is much higher in energy compared to in-phase mode. Fourier transforms of bosonic and fermionic correlation functions, $\left\langle n_{b}(k)\right\rangle=\int \phi_{k}(x) G_{b}(x) d x$ and $\left\langle n_{f}(k)\right\rangle=\int \phi_{k}(x) G_{f}(x) d x$, are given by momentum distributions, shown in Fig. 4. They can be measured using ballistic time-of-flight experiments, since during ballistic expansion momentum distributions get mapped into real space densities [5]. The momentum distribution of composite particles $\left\langle n_{b f}(k)\right\rangle=\int \phi_{k}(x)\left\langle\Psi_{b}^{\dagger}(x) \Psi_{f}^{\dagger}(x) \Psi_{f}(0) \Psi_{b}(0)\right\rangle d x$ has a strong Fermi step, and can be written as $\left\langle n_{b f}(k)\right\rangle=\int\left\langle n_{b}(k-p) n_{f}(p)\right\rangle d p /(2 \pi)$. We note that it is different from $\int\left\langle n_{b}(k-p)\right\rangle\left\langle n_{f}(p)\right\rangle d p /(2 \pi)$, thus the presence of the "pairing" results in nontrivial noise correlations in time-of-flight images [28]. Finally, we would also like to point out the method to measure the correlation functions based on interference of two independent 1D clouds [29, 30]. For bosons (fermions) the average of the square of interference signal $\left\langle\left|A_{b(f)}(L)\right|^{2}\right\rangle$ of two segments of length $L$ is related to an integral of the Green's function as $\left\langle\left|A_{b(f)}(L)\right|^{2}\right\rangle=\int_{0}^{L} \int_{0}^{L} d x d y G_{b(f)}(x-y)^{2}$. The interference signal appears at wave vectors $Q_{b(f)}$, which depend on masses of interfering particles. If one measures $\left\langle\left|A_{b(f)}(L)\right|^{2}\right\rangle$ as a function of $L$, then in principle dependence of $G_{b(f)}(x)$ on distance $x$ can be extracted, since $G_{b(f)}(L)^{2}=\frac{1}{2} \frac{\partial^{2}\left\langle\left|A_{b(f)}(L)\right|^{2}\right\rangle}{\partial L^{2}}$. The same technique can be used also to measure $G_{b f}(x)$, but in this case the information will be contained in the oscillations of the product of Bose and Fermi densities at wave vector $Q_{b}+Q_{f}$. Since in "paired" phase $G_{b f}(x)$ decays much slower with distance than $G_{b}(x) G_{f}(x)$, "paired" phase will be characterized by strong correlations in the fluctuations of bosonic and fermionic interference fringes.

To summarize, we have considered one-dimensional Bose-Fermi mixture with equal densities and unequal masses using DMRG. For the mass ratio of K-Rb mixture and attraction between bosons and fermions, we determined the phase diagram, which is shown in Fig. 1. For weak boson-boson interactions, there is a direct transition between two-component Luttinger liquid and collapsed phases as the boson-fermion attraction is increased. For strong enough boson-boson interactions, we find an intermediate "paired" phase, which is a single-component Luttinger liquid of composite particles. We investigated correlation functions of such a "paired" phase, studied its stability to density imbalance, and discussed various experimental techniques which can be used to detect it.

We thank E. Demler, V. Gritsev, R. Fazio, and F. Dolcini for useful discussions. This work has been developed using the DMRG code released within the "Powder with Power" project (www.qti.sns.it)

[1] S. L. Cornish, et al., Phys. Rev. Lett. 85, 1795 (2000); S. Inouye, et al., Phys. Rev. Lett. 93, 183201 (2004); F. Ferlaino, et al., Phys. Rev. A 73, 040702(R) (2006); C. A. Stan, et al., Phys. Rev. Lett. 93, 143001 (2004).

[2] D. Jaksch, et al., Phys. Rev. Lett. 81, 3108 (1998).

[3] M. Greiner, et al., Nature 415, 39(2002).

[4] T. Kinoshita, T. Wenger and D.S. Weiss, Science 305, 1125 (2004).

[5] B. Paredes et al., Nature 429, 277 (2004).

[6] B. DeMarco and D.S. Jin, Science 285, 1703(1999); F. Schreck et al., Phys. Rev. Lett. 87, 080403 (2001); A.G. Truscott et al., Science 291, 2570(2001); G. Modugno et al., Science 297, 2240 (2002); Z. Hadzibabic et al., Phys. Rev. Lett. 88, 160401 (2002); J. Goldwin et al., Phys. Rev. A 70, 021601(R) (2004); G. Roati, et al., Phys. Rev. Lett. 89, 150403 (2002); C. Silber, et al., Phys. Rev. Lett. 95, 170408 (2005). 
[7] K.K. Das, Phys. Rev. Lett. 90, 170403 (2003).

[8] M. A. Cazalilla, and A. F. Ho, Phys. Rev. Lett. 91, 150403 (2003).

[9] L. Mathey, et al., Phys. Rev. Lett. 93, 120404 (2004).

[10] L. Mathey, D.W. Wang, Phys. Rev. A 75, 013612 (2007).

[11] L. Mathey, Phys. Rev. B 75, 144510 (2007).

[12] X. Barillier-Pertuisel, et al., Phys. Rev. A 77, 012115 (2008).

[13] A. Imambekov and E.Demler, Phys. Rev. A 73, 021602(R) (2006) and Ann. Phys. 321, 2390 (2006); M.T. Batchelor, et al., Phys. Rev. A 72, 061603 (2005); H. Frahm and G. Palacios, Phys. Rev. A 72, 061604(R) (2005).

[14] Y. Takeuchi and H. Mori, Phys. Rev. A 72, 063617 (2005) and Int. J. Mod. Phys. B 20, 617 (2006) and J. Phys. Soc. Jap. 743391 (2005).

[15] P. Sengupta, and L.P. Pryadko, Phys. Rev. B 75, 132507 (2007).

[16] L. Pollet, et al., Phys. Rev. Lett. 96, 190402 (2006).

[17] L. Pollet et al., cond-mat/0609604.

[18] K. Günter, et al., Phys. Rev. Lett. 96, 180402 (2006); S. Ospelkaus et al., Phys. Rev. Lett. 96, 180403 (2006); D. B. M. Dickerscheid, et al., Phys. Rev. Lett. 94, 230404 (2005).

[19] B. Kuklov, and B.V. Svistunov, Phys. Rev. Lett. 90, 100401 (2003).

[20] M.Olshanii, Phys. Rev. Lett. 81, 938(1998).

[21] E.H. Lieb and W. Liniger, Phys. Rev. 130, 1605 (1963); E.H. Lieb, ibid. 130, 1616 (1963).

[22] M. A. Cazalilla, Phys. Rev. A 67, 053606 (2003); B. Schmidt, L.I. Plimak, and M. Fleischhauer, Phys. Rev. A 71, 041601(R) (2005).

[23] S.R. White, Phys. Rev. Lett. 69, 2863 (1992); S.R. White, Phys. Rev. B 48, 10345 (1993); U. Schollwöck, Rev. Mod. Phys. 77, 259 (2005); G. De Chiara, M. Rizzi, D. Rossini, S. Montangero, cond-mat/0603842, to be published on J. Comput. Theor. Nanosci.

[24] M. A. Cazalilla, Journal of Physics B: AMOP 37, S1-S47 (2004).

[25] C. Chin et al., Science 305, 1128 (2004).

[26] H. Moritz, et al., Phys. Rev. Lett. 94, 210401 (2005).

[27] D.M. Stamper-Kurn et al., Phys.Rev.Lett. 83, 2876 (1999).

[28] E. Altman, E. Demler, M. D. Lukin, Phys. Rev. A 70, 013603 (2004).

[29] A. Polkovnikov, E. Altman and E. Demler, Proc. Natl. Acad. Sci. USA 103, 6125 (2006).

[30] A. Imambekov, V. Gritsev, E. Demler, arXiv:cond-mat/0703766v1, Proceedings of "Ultracold Fermi gases" Summer School, Varenna, Italy, June 2006. 